\title{
¿Internacionalizar las licenciaturas de la UNAM?
}

\author{
Pablo G. Hofmann, Tomás García-González, \\ Cecilia Montiel Ayometzi y Melchor Sánchez Mendiola
}

\section{Resumen}

Actualmente, algunos de los procesos de relevancia a nivel global son el crecimiento vertiginoso de la población, así como sus requerimientos de recursos y los efectos que esto produce. Asimismo, la interacción de los grupos sociales se ha intensificado y complejizado, por lo que los retos que surgen de esta realidad no son abordables desde una sola disciplina, por un solo profesionista o en una sola región. Los egresados de las instituciones de educación superior (IES) deben tener la capacidad de identificar y abordar los problemas complejos, de impacto regional o global, con una aproximación interdisciplinaria y multicultural. La Universidad Nacional Autónoma de México (UNAM) está obligada a desarrollar en sus estudiantes las aptitudes necesarias para el ejercicio profesional en un contexto complejo y global. Una estrategia para atender esta necesidad es la creación de planes conjuntos con instituciones educativas de vanguardia que ofrezcan reconocimiento oficial de los estudios realizados. El presente trabajo tuvo como objetivo identificar las IEs de vanguardia a nivel mundial, así como el a nálisis de las condiciones institucionales en la unAm para el establecer convenios interinstitucionales, con el fin de ofrecer planes de estudio de licenciatura compartidos, con doble titulación. Mediante la identificación de las IES de vanguardia, la flexibilidad y áreas de conocimiento que componen las estructuras curriculares de la unAM, así como el marco normativo que las rige, fue posible detectar la oportunidad para establecer los vínculos con otras IEs y el sustento Normativo Universitario para ampliar la oferta educativa de la unAm.

Palabras clave: educación superior, educación internacional, programas conjuntos, doble titulación, competencia profesional, interdisciplinar, multicultural.

\section{INTERNATIONAL UNDERGRADUATE STUDIES AT UNAM?}

\begin{abstract}
Accelerated population growth, with its resource requirements and the effects it produces, is a current relevant global phenomenon. Additionally, the interaction among social groups has become tighter and more complex; hence, the challenges that arise from this new reality cannot be approached from a single discipline, by a single professional, or in a restricted location. Therefore, graduates of higher education institutions (HEIS) must be able to identify and analyze complex problems, with a regional or global impact, from an interdisciplinary and multicultural approach. The National Autonomous University of Mexico (UNAM) is responsible to promote the development of the necessary aptitudes in its students in order to perform their professional exercise in a global and complex context. One of the strategies to meet this need is the creation of joint educational programs with top-performing HeIs that offer official recognition of the studies carried out. The present work is aimed to identify the most prestigious Hels internationally, as well as to analyze the institutional conditions at UNAM, for the establishment of interinstitutional agreements in order to offer joint educational programs for undergraduates with a degree in both
\end{abstract}

Recepción: 09/12/2019. Aceptación: 11/06/2020. Dol: http://doi.org/10.22201/cuaieed.16076079e.2020.21.5.13 
institutions. Through the identification of the top international HeIS, the flexibility and knowledge-field content in the unAm curricular structures, as well as the normative framework that regulates them, it was possible to detect the opportunity for the generation of the affiliations with other HEIS, in addition to the regulatory foundations to expand the UNAm educational spectrum.

Keywords: higher education, international education, joint programs, double degree, professional competence, interdisciplinary, multicultural.

Pablo G. Hofmann

phofmann@unam.mx orcid.org/0000-0002-4701-5117

Biólogo de formación inicial en la Facultad de Ciencias de la Universidad Nacional Autónoma de México (UnAm), con los grados de Maestría Ciencias y Doctorado en Ciencias, ambos por la unam. Se he desempeñado como profesor en las cátedras de Biología del Desarrollo y Embriología Humana en la Facultad de Medicina de la unAm desde 1986. Su interés en el proceso enseñanza-aprendizaje se ha reflejado en su gestión como coordinador del programa de Aprendizaje Basado en Problemas para los estudiantes de primer y segundo años de Medicina, coordinador de evaluación para el Departamento de Embriología de la misma Facultad, así como Responsable del área de Ciencias Biológicas, Químicas y de la Salud en la Subdirección de Planes y Programas de Estudio de la Dirección de Desarrollo Educativo e Innovación Curricular de la unam.

\section{Tomás García González}

tgarcia@codeic.unam.mx

Ingeniero Mecánico Electricista, por la Facultad de Ingeniería de la Universidad Nacional Autónoma de México (UnAm). Maestro en Administración de la Tecnología por la unAm. Ha cursado varios diplomados en las áreas de multimedia y educación. Funcionario en áreas de cómputo en la unAm. Profesor en las facultades de Contaduría y Administración, y de Medicina. Consultor externo en los temas de análisis de patentes y bibliométrico.

\section{Cecilia Montiel Ayometzi}

cmontiel@codeic.unam.mx

Licenciada y Maestra en Pedagogía por la unam. Profesora del Colegio de Pedagogía de la Facultad de Filosofía y Letras, unAm. Desarrolla actividades de planeación educativa, diseño curricular y restructuración de programas de estudio en instituciones públicas y privadas de educación superior.

\section{Melchor Sánchez Mendiola}

melchorsm@unam.mx

Titular de la Coordinación de Universidad Abierta, Innovación Educativa y Educación a Distancia (CUAIEed) de la unAm. Doctor en Ciencias, Educación en Ciencias de la Salud por la unAm; maestro en Educación en Profesiones de la Salud por la Universidad de Illinois en Chicago, EUA. Médico Pediatra por la Universidad del Ejército y Fuerza Aérea Mexicanos (UDEFA). Profesor de Carrera Titular C de Tiempo Completo Definitivo en la División de Estudios de Posgrado de la Facultad de Medicina, unam. 


\section{Introducción}

Uno de los fenómenos de mayor relevancia a nivel global, actualmente, es el crecimiento de la población humana a escala local, regional y mundial, con los requerimientos de espacio, agua, alimentos y energéticos, y los efectos que ello produce (deforestación, desechos biológicos e inorgánicos, etcétera). En los últimos 200 años, el número de habitantes humanos en el planeta ha tenido un crecimiento vertiginoso, desde 989.8 millones en 1800, hasta 7,710 millones en 2019 (HYDE Database, 2019, de la Netherlands Environmental Assessment Agency). Con el crecimiento poblacional, la interacción de los grupos sociales se ha ido incrementando y complejizando.

Dependiendo del enfoque con el que se haya tenido la intención de establecer y describir estos vínculos, así como determinar su robustez, se han definido distintos conjuntos de retos globales, que son problemas de atención prioritaria para incidir positivamente en la calidad de vida de los pobladores del planeta entero, actuales y a futuro. Una de estas organizaciones, el Millennium Project, está constituido por 64 nodos (instituciones e individuos) alrededor del mundo, y publica anualmente el reporte del Estado del Futuro, de acuerdo con los resultados del análisis y proyecciones estimadas (Futures Research Methodology 3.0) en los rubros antes mencionados (Glenn et al., 2016; Glenn et al., 2017). Las propuestas de solución a los retos identificados por esta organización son las siguientes:

1. Logro de un desarrollo sustentable y en relación con el cambio climático

2. Acceso al agua limpia (potable) sin conflicto

3. Crecimiento poblacional y balance con los recursos disponibles

4. Democracia genuina y democratización de regímenes autoritarios

5. Mejora en la toma de decisiones al integrar la previsión global

6. Convergencia de las tecnologías de la información y de la comunicación para beneficio de la población mundial

7. Reducción de la brecha pobres-ricos mediante la promoción de economías de mercado éticas

8. Reducción de la amenaza por enfermedades nuevas y reemergentes

9. Promoción de una educación para enfrentar los retos globales

10. Desarrollo de valores compartidos para reducción de conflicto y promoción de la paz

11. Mejora del estado social de la mujer, lo que incide en la mejora de la condición humana

12. Reducción del poder de las redes de crimen organizado

13. Satisfacción segura y eficiente de la demanda energética creciente

14. Promoción de los avances científicos y tecnológicos para beneficio de la humanidad

15. Incorporación de la ética en la toma de decisiones globales 
Tabla 1. Categorías de competencias consideradas como necesarias para el ejercicio profesional y calidad de vida, en el contexto actual y futuro durante el resto del Siglo xxI. Fuente: Binkley, Erstad, Herman, et al., 2012
La globalización puede entenderse como el incremento de la complejidad de los vínculos intergrupales, así como los problemas, producto de las actividades humanas con un impacto global, derivados del crecimiento de los sistemas sociales, y no solamente los procesos dentro de las esferas económica y política. Estos últimos han sido los conceptos más popularmente difundidos como globalización (Sheffield et al., 2013).

Por la complejidad de los niveles de interacción entre los factores derivados del crecimiento poblacional y las actividades humanas en todo el planeta, los retos globales identificados por las distintas organizaciones no son abordables desde puntos de vista de una sola disciplina, por un solo profesionista o en una sola región. Los retos definidos son de esencia altamente compleja y, además de tener repercusiones a nivel global, afectan regiones dentro de las cuales se encuentran grupos sociales de orígenes étnicos y culturales diversos, con necesidades o requerimientos distintos. El ejercicio profesional, entonces, estará basado en las Ilamadas competencias para el siglo XXI, que deberán ser parte fundamental para el diseño curricular en instituciones educativas alrededor del mundo (Binkley et al. 2012; Marope et al., s.f.). Binkley y colaboradores agrupan estas competencias en cuatro ejes conceptuales o categorías (ver tabla 1).

\begin{tabular}{|c|c|c|c|}
\hline Formas de Pensamiento & Formas de Trabajar & $\begin{array}{c}\text { Herramientas para el } \\
\text { Trabajo }\end{array}$ & Viviendo en el Mundo \\
\hline Creatividad e innovación & Comunicación & $\begin{array}{l}\text { Literacidad en } \\
\text { información }\end{array}$ & $\begin{array}{c}\text { Ciudadanía (local y } \\
\text { global) }\end{array}$ \\
\hline $\begin{array}{l}\text { Pensamiento crítico, } \\
\text { solución de problemas y } \\
\text { toma de decisiones }\end{array}$ & \multirow[b]{2}{*}{$\begin{array}{c}\text { Colaboración } \\
\text { (trabajo en equipo) }\end{array}$} & \multirow[b]{2}{*}{$\begin{array}{l}\text { Literacidad en } \\
\text { Tecnologías de } \\
\text { la Información y } \\
\text { Comunicación (TIC) }\end{array}$} & $\begin{array}{l}\text { Vida y carrera } \\
\text { profesional }\end{array}$ \\
\hline $\begin{array}{c}\text { Aprendiendo a aprender, } \\
\text { Metacognición }\end{array}$ & & & $\begin{array}{c}\text { Responsabilidad } \\
\text { personal y social } \\
\text { (incluyendo } \\
\text { consciencia cultural y } \\
\text { competencia) }\end{array}$ \\
\hline
\end{tabular}

Con la idea de generar las competencias necesarias para el ejercicio actual y futuro de las profesiones, es necesario el diseño e implementación de nuevas estrategias educativas, en conjunto con la redefinición y reingeniería de las estructuras curriculares en las instituciones de educación superior.

Es de suma importancia plantear los procesos educativos de manera tal que las competencias ideales, identificadas por las distintas organizaciones alrededor del mundo, sean el objetivo central de los procesos formativos. Lo anterior, en el entendido de que un plan de estudios está compuesto por una serie de elementos, organizados y planificados, con la intención específica de construcción de conocimientos, habilidades, actitudes y valores, que le permitan al egresado tener la capacidad de abordar problemas complejos de impacto global, como integrantes de grupos multidisciplinarios y multiculturales de profesionistas. 
Una forma de favorecer de manera significativa el desarrollo de estas capacidades es mediante la internacionalización de los programas educativos de las instituciones de educación superior (Barragán-Codina y Leal-López, 2013). La ventaja de una doble titulación es el reconocimiento de los estudios realizados en los países de las instituciones participantes en el convenio; además, por supuesto, de una formación profesional en un ambiente multicultural, que facilite el desarrollo de habilidades de comunicación con equipos de trabajo multinacionales e interdisciplinarios, con un abordaje de problemas complejos desde más de una sola aproximación disciplinar y cultural.

La Universidad Nacional Autónoma de México (UNAM), con la intención de seguir cumpliendo con una de sus labores sustantivas, debe adaptar sus métodos y estrategias educativas para proveer a sus egresados con las herramientas necesarias para un ejercicio profesional de excelencia, en un mundo crecientemente globalizado, complejo y dinámico. Considerar la creación de planes conjuntos con instituciones educativas de otros países, con la posibilidad de doble titulación, será un elemento importante para mantener a la máxima casa de estudios a la vanguardia en la oferta de educación de calidad.

Por lo anterior, en el presente trabajo se exponen los resultados de la identificación de las instituciones de educación superior (IES) de vanguardia a nivel internacional, así como el diagnóstico de las condiciones académicas y normativas de la UNAM, con lo que se aporta información relevante para formular y establecer los convenios con IEs de vanguardia, nacionales y de otros países. Esto permitirá la ampliación de la oferta educativa de la Universidad, mediante la implementación de planes de estudio compartidos, con doble titulación.

\section{Método}

\section{Identificación de instituciones de educación superior de vanguardia}

La identificación de las IEs de vanguardia, de interés para la creación de programas conjuntos, con doble titulación, se obtuvo de los datos publicados por cinco organizaciones evaluadoras del desempeño de las universidades a nivel internacional:

- Academic Ranking World University (ARWU)

- Center for World University Ranking (cWuR)

- $\quad$ Qs World University Ranking (Quacquarelli Symonds Limited)

- Times Higher Education - World University Rankings

- Centre for Science and Technology Studies (cwTs) Leiden Ranking Identificación de las ies de vanguardia a nivel mundial

De los 20 listados publicados (un listado anual, de 2015 a 2018, por cada organización evaluadora), se utilizó la posición promedio de las IES en orden ascendente, como las universidades de vanguardia a nivel mundial. 
Identificación de las ies de Vanguardia a nivel internacional por área de conocimiento

De los listados publicados por la Academic Ranking World University (ARWU) y la QS World University Ranking, para los años 2016 y 2018 respectivamente, se calculó la posición promedio de cada una de las instituciones y en orden ascendente se consideraron como universidades de vanguardia en cada área de conocimiento (las áreas de conocimiento se consideran de acuerdo al Artículo 16 del Estatuto General de la Universidad Nacional Autónoma de México, 2019).

Diagnóstico de los planes de estudio de licenciatura de la UNAM, en modalidad presencial

De los planes de estudio a nivel licenciatura en la unAm, impartidos en modalidad presencial, se accedió a la información contenida en el Sistema Integral de Administración Escolar (SIAE) de la Dirección General de Administración Escolar de la unAm (www.dgae-siae.unam.mx), se descargó el listado total de asignaturas, tanto obligatorias como de elección. Del listado total de asignaturas de cada uno de ellos, se estimó el porcentaje de asignaturas obligatorias y asignaturas de elección. Se calculó también el porcentaje o proporción correspondiente a las áreas de conocimiento, según las agrupa la unAm ( a Iv).

Análisis de la Normatividad Universitaria para la creación de programas conjuntos con doble titulación

Con la intención de identificar los elementos normativos que dan sustento para pactar los convenios pertinentes de creación de programas conjuntos, con doble titulación, se realizó una búsqueda dentro de los Documentos de Normatividad Académica de la unam, con los términos "compartidos", "conjuntos", "convenios", "flexibilidad", "movilidad", "titulación" e "internacional".

\section{Resultados y discusión}

Identificación de Instituciones de Educación Superior de Vanguardia a nivel mundial

Con la posición promedio de las 200 Instituciones de Educación Superior (IES) de mejor desempeño, entre 2015 y 2018, se pudo identificar a las 317 de más alto desempeño a nivel internacional (ver gráfica 1, primeras 50 IES). Es notable la predominancia de las que se encuentran en países de habla inglesa (Estados Unidos de América y el Reino Unido, 41\%) en el período mencionado. En el caso de México, la Universidad Nacional Autónoma de México es la única ies que figura, consistentemente, dentro de los resultados de los análisis de las organizaciones evaluadoras, en la posición promedio 186 (ver tabla S1. Material suplementario). 
Gráfica 1. Representación gráfica del rango de ubicación de cada una de las 50 ies de más alto desempeño dentro de las jerarquizaciones publicadas, a nivel internacional, para el período comprendido entre 2015 y 2018. En este gráfico de cajas y bigotes, es posible apreciar la posición más alejada del primer lugar (dato de valor más alto en los bigotes), la posición más cercana a la primera posición (dato de menor valor en los bigotes) y el rango dentro del que más frecuentemente se ubicó a la institución (caja azul). El orden de las instituciones dentro de la gráfica corresponde a la posición promedio (en orden creciente, desde la posición más cercana al primer lugar, hasta la posición 50) en las jerarquizaciones de las 5 organizaciones evaluadoras en cada uno de los años, entre 2015 y 2018 (línea interna en la caja).

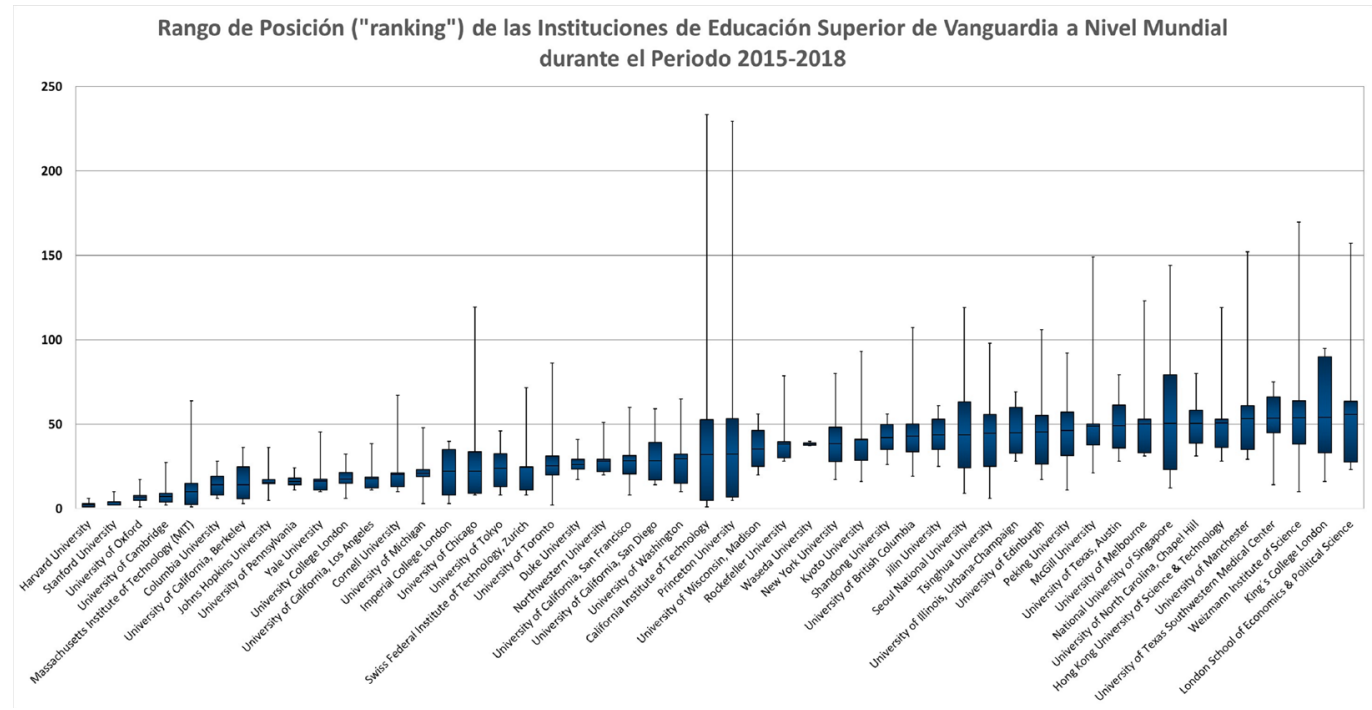

Además del desempeño global por su posición promedio cercana al primer lugar (más alto puntaje en las evaluaciones), una característica de las IES que puede tomarse en cuenta para definirlas como de vanguardia es qué tan constante es su posición dentro de las jerarquizaciones y qué tan amplio el rango de su posición. Esto se puede considerar como un indicador de la estabilidad académica en la realización de sus funciones.

Con las 317 IEs identificadas como de vanguardia en el presente análisis como base, se consultaron las categorizaciones por la ARwu y la Qs World University Ranking, para los años 2016 y 2018, respectivamente, por área de conocimiento (Estatuto General de la Universidad Nacional Autónoma de México, 2019). Para el Área de las Ciencias Físico-Matemáticas y de las Ingenierías se identificaron 126 IES (ver tabla S2. Material suplementario), para las que destacan en temas considerados del Área de Ciencias Biológicas, Químicas y de la Salud, un total de 148 (ver tabla S3. Material suplementario), dentro del Área de Ciencias Sociales, un total de 122 Universidades (ver tabla S4. Material suplementario) y finalmente 49 IES que mejor se desempeñan en temas que corresponden al área de las Humanidades y de las Artes (ver tabla S5. Material suplementario).

Se hizo evidente que las instituciones educativas que ocupan las posiciones más cercanas al primer lugar son las que más frecuentemente se presentan en los listados, pero, además, el rango de su ubicación dentro de las jerarquizaciones es menos variable. Esto quiere decir que, sin importar de qué manera se evalúen, los indicadores considerados y los elementos que componen a cada indicador, así como la ponderación que los indicadores tengan dentro de cada evaluación global, las IES de mejor desempeño académico se mantienen siempre en los primeros lugares.

Es importante hacer una reflexión respecto a los principios bajo los cuales estas IES, que se encuentran siempre entre los primeros lugares, efectúan sus labores educativas, la generación de conocimiento, la difusión del mismo y su 
impacto a nivel global. Una revisión de la filosofía de trabajo y los mecanismos administrativos, más que los contenidos en los programas de estudio de éstas Instituciones, es de suma relevancia para que la unAm considere el establecimiento de convenios académicos estratégicos y que en éstos se plantee la existencia de proyectos educativos conjuntos, con enfoques multiculturales, multi, inter y transdisciplinarios, con la emisión de un título o diploma por ambas instituciones.

\section{Diagnóstico de los planes de estudio de licenciatura de la UNAM, en modalidad presencial}

Se identificaron 126 planes de estudio de nivel licenciatura en modalidad presencial, de los cuales 37 corresponden al Área de las Ciencias FísicoMatemáticas y de las Ingenierías (área I), 27 al Área de las Ciencias Biológicas, Químicas y de la Salud (área ॥), 22 al Área de las Ciencias Sociales (área ॥I) y 40 al Área de las Humanidades y las Artes.

El cálculo de la proporción de los elementos curriculares de elección, respecto a aquellos de carácter obligatorio, hizo evidente el grado de flexibilidad de los planes de estudio de las cuatro áreas de conocimiento en la unAm. De igual manera que para la flexibilidad, se agrupó a las asignaturas por área de conocimiento (áreas ı a ıv), implicadas en cada plan de estudios, con base en su denominación y contenidos, obteniendo la proporción de cada una de éstas (ver gráficas S1, S2, S3 y S4. Material suplementario).

La proporción de los elementos curriculares de elección, o de las áreas de conocimiento, para cada plan de estudios, se podría considerar como la parte formativa cursada en el plan de estudios de una IES diferente a la UNAM, para establecer el convenio correspondiente. Mediante la realización de estudios superiores en contextos internacionales, se fomenta el desarrollo de vínculos y comprensión de otras culturas, así como la capacidad de comunicación efectiva en más de un solo idioma, y, con esto, se incide en la formación profesional con el desarrollo de las competencias descritas para el desempeño profesional durante el resto del siglo xx. Esta información, en conjunto con la jerarquización de las ies que destacan por su desempeño en las diferentes áreas de conocimiento, sirve como base para realizar un análisis más detallado y que los cuerpos colegiados en las entidades académicas de la UNAM consideren la posibilidad del establecimiento de los convenios pertinentes, para la obtención del reconocimiento oficial o título emitido por las dos instituciones.

\section{Análisis de la Normatividad Universitaria para la creación de programas conjuntos con doble titulación}

La Normatividad Universitaria vigente, al momento de la redacción de este informe, contiene diversos artículos que mencionan los conceptos de planes compartidos, planes conjuntos, convenios, flexibilidad, movilidad, titulación e internacionalización. Aun cuando dichos artículos no hacen mención de los 
Tabla 2. Normatividad Universitaria vigente que da sustento legal para la creación de planes o programas conjuntos, con doble titulación, previo convenio establecido con instituciones de educación superior de vanguardia. Fuente:

Legislación Universitaria de la Universidad Nacional Autónoma de México.

mecanismos por los cuales se pueden materializar los convenios para programas conjuntos, con doble titulación, el Marco Institucional de Docencia (UNAm, 2003) en su Artículo 12, hace mención de que el valor en créditos de una asignatura, módulo o práctica obligatoria (unidad curricular) no podrá ser sustituido por el valor de otra unidad curricular, obligatoria u optativa, a menos que se explicite esta condición en el plan de estudios, o esta situación sea dictaminada por el Consejo Técnico correspondiente. De la misma manera, los Lineamientos Generales para el Funcionamiento de los Estudios de Licenciatura (UNAM, 2015), en el numeral xIx del Artículo $8^{\circ}$, así como los Lineamientos Generales para el Funcionamiento del Posgrado (UNAM, 2007), en su Artículo $2^{\circ}$, estipulan que es atribución de los Consejos Técnicos de las Entidades, o de los Comités Académicos de los Estudios de Posgrado, respectivamente, autorizar la sustitución de las actividades obligatorias u optativas por otras del mismo plan u otros planes vigentes (ver tabla 2). Con base en estos fundamentos normativos, es posible precisar las bases para establecer los convenios con otras IEs, en los que se defina explícitamente que una proporción específica (unidades curriculares o créditos) de la carga académica (equivalente y equitativa) podrá ser cursada en la institución partícipe del convenio para que ambas instituciones emitan un título.

Documento normativo

Marco institucional
de docencia (2003)

Ineamientos generales para el funcionamiento de los estudios de licenciatura (2015)

Lineamientos
generales para el
funcionamiento del
posgrado (2007)

\section{Artículo}

Artículo 12. El valor en créditos de una asignatura, módulo, curso o práctica obligatoria, no podrá sustituirse por el valor de otra asignatura, módulo, curso o práctica, sea obligatoria u optativa, a no ser que consten explícitamente en el plan de estudios los casos de excepción, o éstos sean dictaminados por el consejo técnico correspondiente.

Artículo $8^{\circ}$. Para las licenciaturas disciplinarias, de conformidad con la normatividad vigente, los consejos técnicos de las facultades y escuelas tendrán, al menos, las atribuciones siguientes:

xIx. La sustitución de actividades académicas obligatorias y optativas de un plan de estudios, por otras del propio plan o de otros planes vigentes;

Artículo $2^{\circ}$.Las actividades académicas obligatorias de un plan de estudios podrán ser sustituidas por otras actividades académicas, obligatorias u optativas, del propio plan o de otros planes vigentes, de acuerdo con lo establecido en el Marco Institucional de Docencia, previa autorización del comité académico o del cuerpo colegiado encargado de la conducción del programa para el caso de las especializaciones.

\section{Conclusión}

La internacionalización de la educación superior es, en gran medida, una respuesta a las demandas de desarrollo de conocimientos, habilidades, actitudes y valores para el ejercicio profesional en todas las esferas del quehacer humano, dentro de un ambiente complejo y altamente dinámico a escala mundial. El caso específico de la creación de programas educativos en conjunto con instituciones de educación superior a nivel internacional es de importancia insoslayable para promover el desarrollo de la capacidad de funcionar dentro de grupos de trabajo multiculturales y multidisciplinarios, con habilidades de comunicación oral y escrita en más de un idioma, además de proporcionar la facultad para observar 
la realidad (local, regional o global) desde múltiples puntos de vista. Dentro del contexto de los retos globales, definidos de manera consensada por diferentes organizaciones, los procesos educativos deben estar orientados a la preparación de individuos capaces de identificar los problemas, definir su complejidad, así como de proponer distintas aproximaciones y las posibles soluciones, con plena consciencia de su repercusión en los ámbitos social, económico y ambiental.

Derivado del estudio realizado, es posible apreciar la ventana de oportunidad para establecer los vínculos con instituciones de educación superior y ampliar la oferta educativa de la Universidad Nacional Autónoma de México. Especialmente, con la mira puesta en la internacionalización de los planes y programas de estudio. Para este fin, es necesario hacer del conocimiento de los cuerpos colegiados de las entidades académicas de la unAm, la filosofía y ventajas de la apertura y flexibilización de los procesos de formación. Todo esto con la intención de ampliar las fronteras del ejercicio profesional, no sólo en términos geográficos, sino también en la extensión de las herramientas cognitivas y competencias para la inserción de los egresados a un mundo globalizado, complejo y dinámico.

La información presentada en este documento, más que trazar una línea de acción, pone sobre la mesa las opciones para que cada entidad, de acuerdo con su juicio, considere la posibilidad de crear los programas conjuntos pertinentes y factibles, que estén plenamente justificados académicamente. Mediante la aprobación de los Consejos Técnicos de las entidades responsables de los planes de licenciaturas disciplinarias, así como de los Comités Académicos encargados de la conducción de los planes de licenciaturas interdisciplinarias, o de programas de posgrado, los estudios conjuntos realizados con doble titulación o grado podrán ser parte de la oferta educativa de la Universidad Nacional Autónoma de México.

Por todo lo expuesto anteriormente, se hace patente la posibilidad de crear planes de estudio compartidos o conjuntos entre la UNAM y otras IES, en los niveles nacional, regional y mundial, con la intención de promover una formación más acorde a las necesidades emergentes de una realidad global.

\section{Referencias}

* Barragán-Codina, J. N. y Leal-López, R. H. (2013, agosto). The Importance of Student Mobility, Academic Exchange and Internationalization of Higher Education for College Students in a Globalized World. Daena: International Journal of Good Conscience, 8(2):48-63. http://www.spentamexico.org/v8-n2/A3.8(2)48-63.pdf

* Binkley, M., Erstad, O., Herman, J., Raizen, S., Ripley, M. Miller-Ricci, M. y Rumble M. (2012). Defining Twenty-First Century Skills. En P. Griffin, B. McGaw y E. Care (Eds), Assessment and teaching of 21st century skills. Springer. Dol: https://doi. org/10.1007/978-94-007-2324-5_2 
* Glenn, J. C., Florescu, E. y Millennium Project Team. (2016). 2015-16 State of the Future.Journal of Socialomics, 5(3):1000168. Dol: https://www.doi.org/10.4172/21670358.1000168

* Glenn, J. C., Florescu, E. y Millennium Project Team. (2017). Executive Summary. En State of the Future 19.0. Library of Congress. http://www.millennium-project.org/ state-of-the-future-version-19-0/

* Marope, M., Griffin, P. y Gallagher, C. (sin fecha). Future Competences and the Future of Curriculum. International Bureau of Education (IBE), United Nations Educational, Scientific and Cultural Organization (unEsCo). http://www.ibe.unesco. org/sites/default/files/resources/future_competences_and_the_future_of_ curriculum.pdf

* Sheffield, J., Korotayev, A. y Grinin, L. (2013). Globalization as a link between the past and the future. En J. Sheffield, A. Korotayev y L. Grinin (Eds.), Globalization: yesterday, today and tomorrow (pp. 2-29). Emergent Publications. USA.

\section{Bases de datos consultadas}

- Academic Ranking World University (arwu)

- Centre for Science and Technology Studies (cwts) Leiden Ranking

- Center for World University Ranking (cwur)

- $\quad$ hyde Database (2019) Netherlands Environmental Assessment Agency

- $\quad$ Our World in Data

- $\quad$ qs World University Ranking (Quacquarelli Symonds Limited)

- Sistema Integral de Administración Escolar (Dirección General de Administración Escolar de la unam

- Times Higher Education - World University Rankings

\section{Normatividad Universitaria revisada}

Disponible en: http://abogadogeneral.unam.mx/legislacion/consulta/\#

- $\quad$ unam (2019) Estatuto General de la Universidad Nacional Autónoma de México

- $\quad$ unam (2003) Marco Institucional de Docencia

- $\quad$ unam (2014) Reglamento General de Estudios Universitarios

- $\quad$ unam (2018) Reglamento General de Estudios de Posgrado

- unam (2015) Reglamento General para la Presentación, Aprobación, Evaluación y Modificación de Planes de Estudio

- $\quad$ unam (2009) Estatuto del Sistema de Universidad Abierta y Educación a Distancia 
- $\quad$ unam (2015) Lineamientos Generales para el Funcionamiento de los Estudios de Licenciatura

- $\quad$ unam (2007) Lineamientos Generales para el Funcionamiento de Posgrado

\section{Cómo CITAR ESTE ARTículo}

* Hofmann, Pablo G., García-González, Tomás, Montiel Ayometzi, Cecilia y Sánchez Mendiola, Melchor (2020, septiembre-octubre). ¿Internacionalizar las licenciaturas de la UnAm? Revista Digital Universitaria (RDU), 21(5). Dol: http://doi.org/10.22201/ cuaieed.16076079e.2020.21.5.13 